\title{
Simulation based Design of Innovative Quick Response Processes in Cloud Supply Chain Management

\author{
for "Slow Food" Distribution
}

\author{
Agostino G. Bruzzone, Marina Massei, Francesco Longo, Davide Scalzo, Carlo Martini, \\ Jonathan Villanueva, Luca Bucchianica \\ Simulation Team \\ Email \{agostino.bruzzone, marina.massei, francesco.longo, davide.scalzo, carlo.martini, \\ jonathan.villanueva, luca.bucchianica\}@simulationteam.com \\ URL www.simulationteam.com
}

\begin{abstract}
This paper proposes an innovative business model for making accessible premium quality food worldwide, in respect with its origins and cultural background; the authors present a simulation approach to design the general architecture and the supply chain processes devoted to achieve this result as well as the description of the Supply Chain Architecture. The authors introduced the concept of data consistency for processing reliability of the input over the uncertainty of market demand as well as the influence of stochastic factors. The paper proposes this case study as a good example of using these innovative techniques integrated with simulation.
\end{abstract}

Keywords: Cloud, Supply Chain Management, Simulation

\section{Introduction}

The design of a new supply chain is always a very difficult problem considering that is affected by many stochastic elements (e.g. delays in delivery, fluctuations in custom fees, supplier and production reliability, customer satisfaction, demand evolution); therefore when the supply chain addresses new business sectors and markets the problems is even more complex considering the uncertainty related to the capability to create a consistent demand. In this sense the idea to develop a slow food culture, related to premium quality food, within new countries is a very good example for this kind of 
challenges. Slow food represents indeed the reaction to "fast food" approach and consider critical to develop a culture of food and a respect for high quality alimentary products related to typical traditions of different areas and regions; this concept was introduced probably around middle of ' 80 in Italy and France and promoted the establishment of networks of producers and restaurateurs respecting the original quality of the food (Portinari 1989; Petrini 2003, 2013).

Most recently the World Expo in Milan was exactly focused on food and importance of guarantee quality and to feed the whole planet (Cull 2015). For instance the cheese market in China was mostly not existing till few years ago and several companies trying to import there goods from France or Italy achieved very bad results (Debuef et al. 2004); therefore the world is evolving and globalization deals also with blending different cultures and diffusing new knowledge on emerging areas. The authors are currently interested in developing processes to distribute high quality food products worldwide and especially in Far East, by delivering not only the goods, but even the cultural background related to them. This process deals with creating kits to be available for customers worldwide by web service and, concurrently, to connect the qualified original producers directly through cloud service. This paper presents a preliminary approach to develop a simulation model of the supply chain management (SCM) reproducing this processes and including information and good flows; therefore in this case it becomes critical to consider that the input data are strongly affected by hypotheses about market reactions. From this point of view it is proposed an innovative approach in characterizing the input not only in terms of values, but also in terms of their confidence band and reliability of the info and sources (Bruzzone et al. 2008; De Felice et al. 2010); these variables are used by the simulator producing results that could estimate the consistency of the results, the experimental error of the models as well as the overall reliability of the output.

\section{Cloud Manufacturing (CMfg) for Slow Food SCM}

CMfg is a new, production networks-based paradigm that enables to increase readiness and capabilities of the whole network (Tao et al.2011). The CMfg provides a safe, reliable, high quality, on demand and economic service for the entire production life cycle (Ren et al. 2014). 


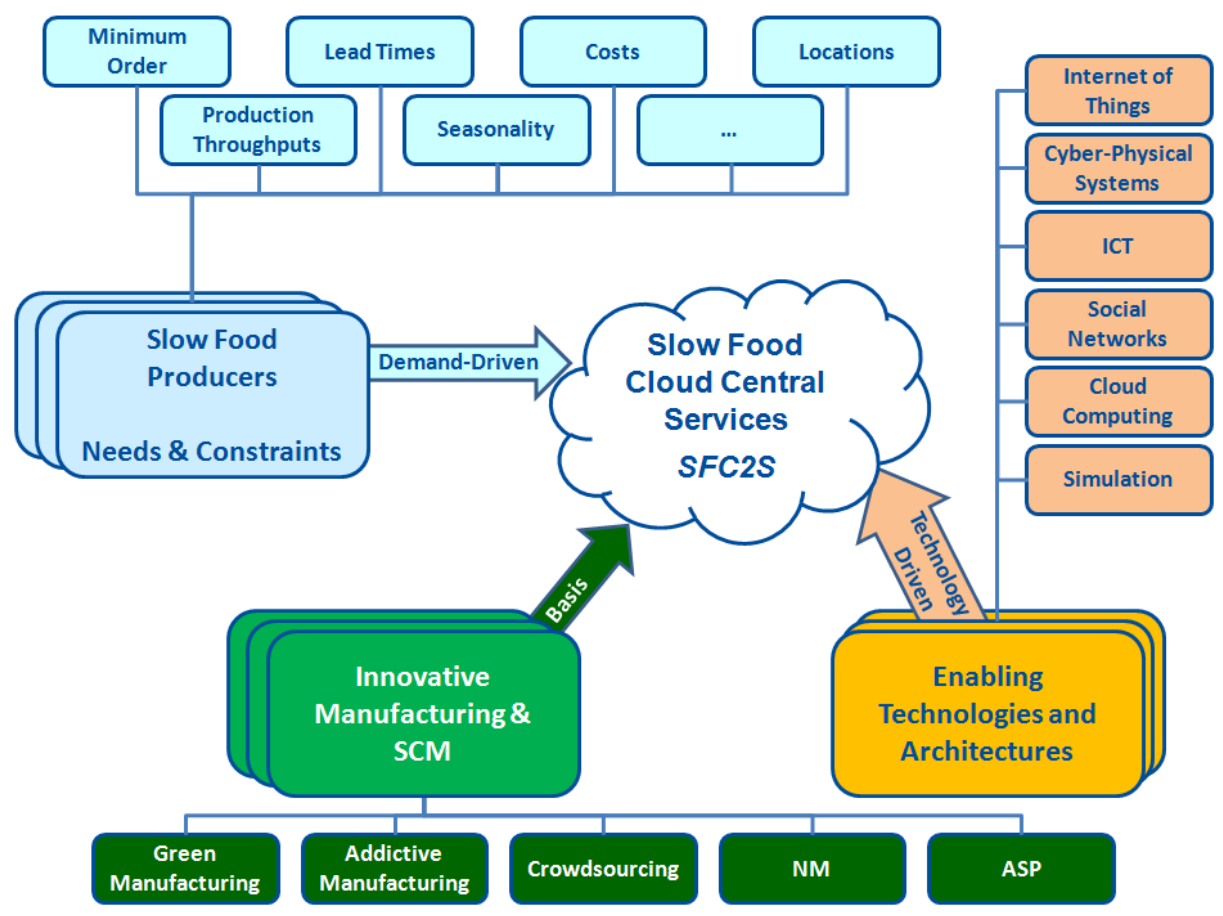

Fig. 1. CMfg applied to Slow Food Cloud Central Services

Being the case study focused on slow so high quality products and not off-the-shelf goods, the authors emphasize the importance of agility for CMfg, requiring an high degree of capability to be adaptive to evolution of market conditions and changes in customer requirements (Hao et al. 2005; Wu et al. 2013; Tao et al. 2014). The enabling factors for Cloud Manufacturing and SCM are summarized in figure 1 for our context in consistency with existing research in other areas (Zhang et al. 2010a). The maximum degree of adaptability has to be reached through five forms of flexibility respect the Resource Service Composition (RSC): Task, Flow, Resource Service, Quality of Service (QoS) and Correlation (Zhang et al. 2010b). The purpose of such platform is providing common features to support customers and suppliers to achieve their goals, therefore it is necessary to adopt a proper business model, able to generate added value for all the "actors" involved in order to be widely used for cooperation and collaboration purposes (Bruzzone et al. 2012). The use of a core engine to distribute the orders and to decide delivery process and routing guarantees a continuous improvement (Baruwa et al.2008; Vonolfen et al.2011). Existing researches in this sector outline the fact for collaboration it is intended the capability of several actors to work together to 
gain common results by sharing knowledge and expertise (Parker 2007). When actors share information an Intellectual Property Right (IPR) issue could arises. It mainly focuses on the use and control of background and foreground rights and could be addressed and solved only through effective negotiation among the actors. Starting from these considerations, the authors propose their vision on slow food SCM by using CMfg as a strategic "tool" able to support future globalized food chain with respect of its original high quality characteristics.

\section{Case study: "Eat in Cloud"}

The case study proposed in this paper, as anticipated. is related to the model of a new business titled "Eat in Cloud". In facts the basic concept of "Eat in Cloud" comes out combining two basic principles: the first is to provide consumers with high quality and certified products immersed within their cultural heritage and the second is to help and facilitate cooperation among SMEs (Small Medium Size Enterprises) active in agricultural business such as Olive Oil Producers, Gourmet artisans (Jüttner et al. 2009). The complexity of this supply chain suggests to adopt M\&S as main investigation technique (Busato et al. 2009). Therefore the "Eat in Cloud" goal is to propose itself as a powerful service concurrently to SMEs and consumers, using Internet and its ability to create social dynamic networks and to combine different technologies; most of these products result fresh and strongly affected by logistics processes, so these elements make it evident the necessity to use M\&S (Modeling and Simulation) as a tool to reproduce the related processes (Bruzzone, Massei and Bocca 2009); indeed the coordination network in some way is mirroring the concepts in use for "Cloud Computing" with benefits of evolving Internet of Things (IoT) that provide quick and immediate access to these services as well as to control the related processes creating useful synergies such as catching the proper vector for delivery at a specific destination in respect of transportation constraints (Rodrigue et al. 2006; Rossi et al 2012). The processes in order to be able to respect time and cost constraints, as well as the handling protocols that guarantee organoleptic characteristics of the food, need to develop effective management algorithms (Sun et al. 2005; Bruzzone et al.2013). Obviously the success of this initiative is deeply related to the capability to identify the proper ways to penetrate the region and to create this new market; this deals with the identification of target customers (e.g. customer profile in terms of culture, social status, age) and specific area to address (e.g. most promising countries, provinces, 
towns); for instance it is crucial to define if it makes sense to start promotion through service to regional restaurants within an area or to address special events or directly the final customers; obviously these alternatives are going concurrently, therefore it should be tuned the effort for each single channel (Bruzzone et al. 2004). Due to these reasons it becomes evident the necessity to collect the large quantity of available data on this framework and to adopt most recent analysis and management techniques for processing these big data.

In similar way, it necessary to proceed to identify and promote the initiative among SME's for their development and growth. Indeed this requires the capability to react dynamically, in full respect of the original and traditional food production processes, to the demand by adopting a quick response delivery service guaranteeing the high quality of the products. Marketing will be also important to guarantee the flows needed for the SCM, therefore the quality of the service obviously relies on the capacity to include in the delivery kits also the value of the food cultural heritage. For instance the delivery of a specific slow food related to the region should include additional info, pictures, video, image, crafts and elements allowing to perceiving properly this good within his cultural framework.

The model proposed by "Eat in Cloud" is based on reliability and collaboration among the cloud service providers and slow food producers; in this way it results possible to advance in creating an efficient logistic and distribution model avoiding wastes and guaranteeing a smart and tailored service for each single consumer and channel (Massei 2006); this approach could lead to improve the whole supply chain and to support evolution of SME (Merkuryev et al. 2008, 2009; Merkuryeva et al. 2011).

\section{$4 \quad$ Logistics Architecture}

It is hereafter proposed the preliminary logistics model adopted for investigating this context; the approach is based on a service model coordinated by a Slow Food Cloud Central Services (SFC2S) that regulates orders and deliveries among producers and customers.

In facts based on agreements among food producers and SFC2S, the goods are expected to be stocked in the nearest warehouse available in consistent quantities; in this location the kits are prepared by combining the different elements; the number of kits to be available in the warehouses are estimated based on data fusion predictive analysis. The forecasts consider historical sales, measured trends and market 
expectations, the values are corrected in consistency with sustainable volumes in terms of logistics costs, capabilities and constraints (Pfohl et al. 2010).

It is proposed an architecture based on a logistics network composed by a set of distributed hub covering different regions to be served both in the customer area and in production sites; therefore it is necessary to evaluate dynamically for each good the trends for country and target customers and to aggregate them in each hub in relation to the logistics network architecture and flows.

As consequences of this analysis the management algorithms are devoted to achieve several main goals including, respect of food protocols, time response with minimum stocks in each warehouse, reduction of unsold goods stocked through pre-assignments, based on preventive analysis, and promotional distribution policies to affect the demand.

A simple representation of the logistics is proposed in the figure 2 and considers the different delivery approaches:

- One Step Deliveries, further subdivided into the following elements:

- Producer $\rightarrow \quad$ Slow Food Area Hub

- Slow Food Area Hub

$\rightarrow$ Consumer

-Two Step Deliveries devoted to regulate the following elements:

- Producer $\rightarrow$ Slow Food Area Hub

- Slow Food Area Hub $\rightarrow$ Customer Regional Hub

- Customer Regional Hub $\rightarrow$ Consumers

Talking about logistic hubs, we refer to a service like WaaS (Warehouse as a Service), also known as a temporary warehouse service; in this way the platform decreases the fixed costs and guarantees dynamic and scalable services enhancing the agility of the SCM.

\section{Modeling the Processes and Evaluating the Data Consistency}

As previously said, the "Eat in Cloud" concept is not limited to sales and distribution only, but includes also elements of the production processes as in other industrial context (Macias et al. 2004). As matter of fact, often SME's in agricultural business, are not optimizing their production processes and/or waste their assets; in this context the assets are not limited to primary goods, but include also their production capability, their resources and their know-how. 


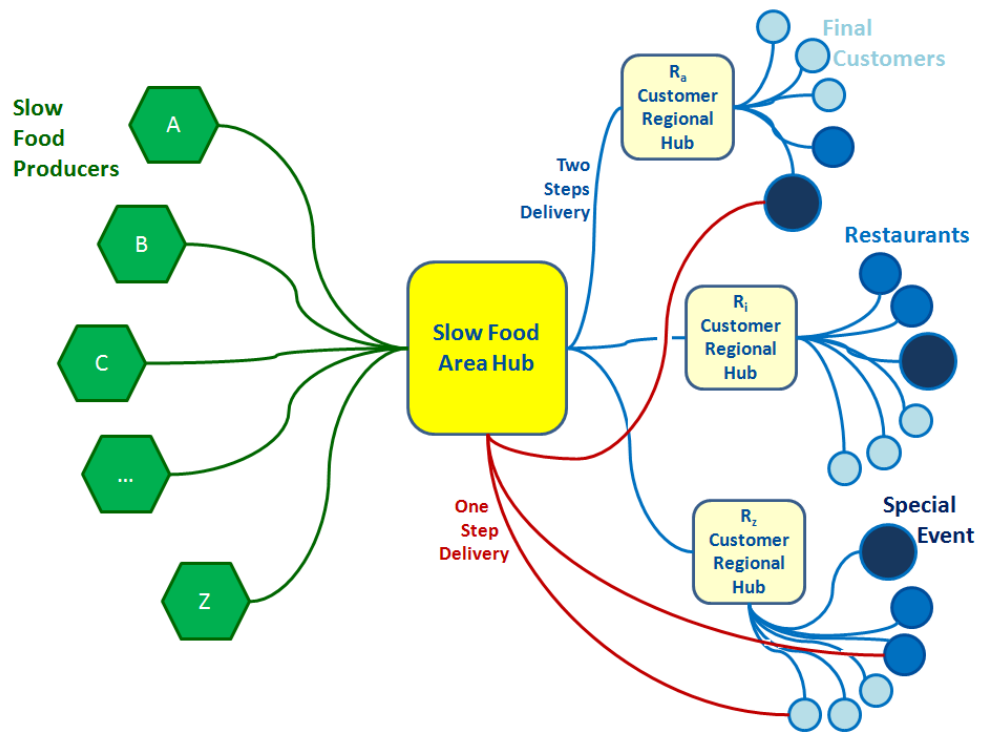

Fig. 2. Slow Food Supply Chain with different delivery approaches

The general scheme of the delivery process is summarized in the figure 2 . Therefore the intent of the proposed SFC2S platform is to avoid, or at least mitigate the asset waste, creating and reinforcing the interaction among the productive realities by establishing virtual enterprises able to react dynamically to the demand evolution and to manage new kind of products obtained by combining their primary goods with other ones and/or with additional cultural heritage elements; in this way it is possible not only to maintain, but also to raise the qualitative standards which are the base of our productive vision and to penetrate new market by developing high value goods.

Obviously to estimate the market response requires to develop models not only of the production, but also of the markets; it is also crucial to be able to evaluate the reliability of the SCM as well as the confidence of the estimations and hypothesis provided by the experts on a specific area or region and could support evaluation of the supply chain reliability (Christopher et al. 2004; Sceffi 2005; Sceffi et al. 2005; Longo et al. 2008; Falasca et al. 2008; Barroso et al. 2011; Stravos 2012). These assessments could be obtained through analysis on the big data by applying data farming and design of experiments (Montgomery 2000). A representation of the connections among the different entities is proposed in the figure 3 ; the lead times and specific costs affect the customer satisfaction during the simulation therefore also the general market situation is used as a bias for considering the performance of our new cloud services; this 
approach is an evolution of previous models used to represent fresh food supply chain (De Sensi et al. 2008; Bruzzone et al.2013). The SFC2S platform will serve as a slow food pool, where each good, defined in terms of quality, quantity and readiness, will be defined by each producer. A common matching algorithm engine will act as core engine for SFC2S platform to suggest and notify new opportunities for possible developments and synergies among goods and producers.

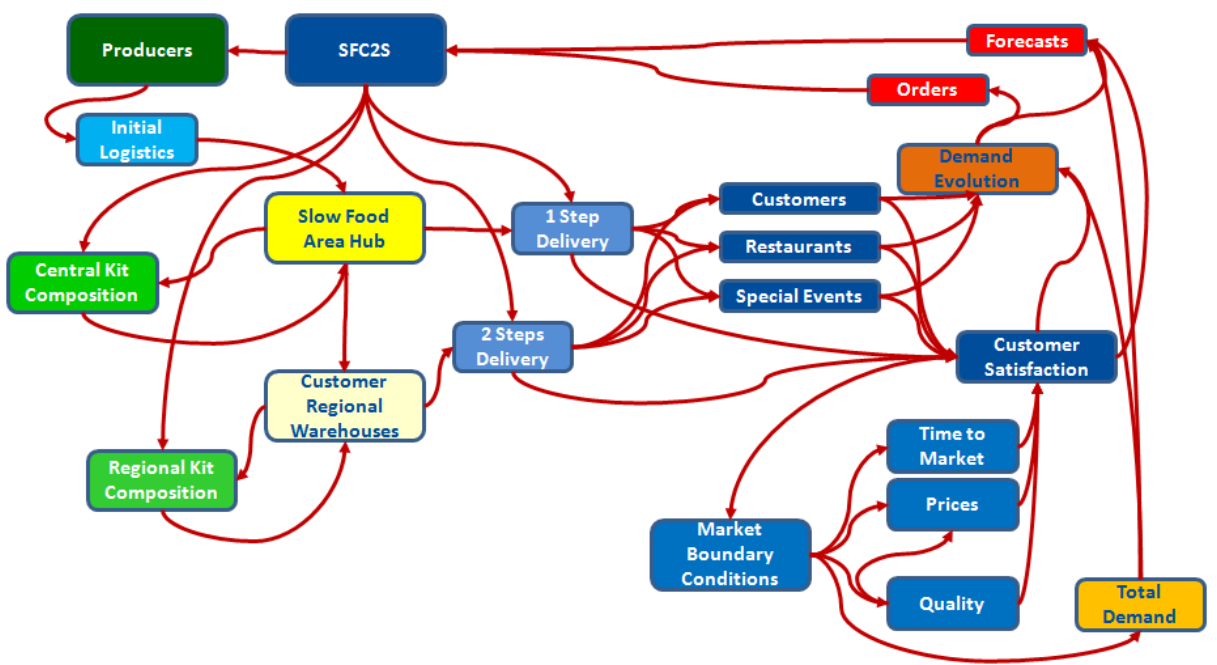

Fig. 3. Interaction among the different entities of the SCM

The algorithm should consider the reliability and confidence of the estimations; these parameters and their trends and standard deviations could be computed based on forecasts and orders dynamic evolution; indeed the data consistency could evaluated by the following functions:

$$
\begin{gathered}
A o^{j}\left(t, t_{0}\right)=\sum_{i=1}^{n^{j}} H\left(O D D^{j}{ }_{i}(t)-t_{0}\right) \cdot H\left(t_{0}+I t-O D D^{j}{ }_{i}(t)\right) \cdot O S^{j}{ }_{i}(t) \\
F o^{j}\left(t, t_{0}, d p t\right)=A o^{j}(t-d p t)+\int_{t_{0}}^{t_{o+I t}} F S^{j}(t, t-d p t) d t \\
D f^{j}\left(t, t_{0}, d p t\right)=A o^{j}\left(t_{0}+I t, t_{0}\right)-F o^{j}\left(t, t_{0}, d p t\right) \\
D a^{j}\left(t, t_{0}, d p t\right)=A o^{j}\left(t_{0}+I t, t_{0}\right)-A o^{j}\left(t, t_{0}\right)
\end{gathered}
$$

$\operatorname{Ao}^{\mathrm{j}}\left(\mathrm{t}, \mathrm{t}_{0}\right) \quad$ Actual Orders for $\mathrm{j}$-th product type at $\mathrm{t}$ time expected to be delivered between $t_{0}$ and $t_{0}+$ It 


\begin{tabular}{|c|c|}
\hline $\mathrm{Fo}^{\mathrm{j}}\left(\mathrm{t}, \mathrm{t}_{0}, \mathrm{dpt}\right)$ & $\begin{array}{l}\text { Future Order Estimator for } j \text {-th product type at } t \text {-dpt time, } \\
\text { expected to be delivered between } t_{o} \text { and } t_{0}+I t\end{array}$ \\
\hline $\mathrm{Fs}^{\mathrm{j}}\left(\mathrm{t}, \mathrm{t}_{0}, \mathrm{dpt}\right)$ & $\begin{array}{l}\text { Forecasts of quantities to be delivered at } t \text { time, net from } \\
\text { consolidated Orders, for } j \text {-th product estimated at } t \text {-dpt time }\end{array}$ \\
\hline dpt & Temporal anticipation for evaluating future orders \\
\hline $\mathrm{n}^{\mathrm{j}}$ & Number of orders for $j$-th product type \\
\hline $\mathrm{t}$ & Time \\
\hline $\mathrm{t}_{0}$ & Beginning of the time interval for order consistency evaluation \\
\hline It & Time Interval for Order Consistency Evaluation \\
\hline $\mathrm{ODD}_{\mathrm{i}}(\mathrm{t})$ & Delivery Date of $\mathrm{i}$-th order for $\mathrm{j}$-th product type at $\mathrm{t}$ time \\
\hline $\mathrm{OS}_{\mathrm{i}}(\mathrm{t})$ & Status of $\mathrm{i}$-th order for $\mathrm{j}$-th product type at $\mathrm{t}$ time [ 0 inactive, 1 active] \\
\hline $\mathrm{H}(\mathrm{x})$ & Heavyside function \\
\hline $\operatorname{Df}^{j}\left(t, t_{0}, d p t\right)$ & $\begin{array}{l}\text { Difference between the real finalized orders within } t_{0} \text { and } t_{0}+\mathrm{It} \\
\text { and the Forecasts at } t \text {-dpt time for } j \text {-th product }\end{array}$ \\
\hline $\mathrm{Da}^{\mathrm{j}}\left(\mathrm{t}, \mathrm{t}_{0}, \mathrm{dpt}\right)$ & $\begin{array}{l}\text { Difference between the real finalized orders within } \mathrm{t}_{0} \text { and } \mathrm{t}_{0}+\mathrm{It} \\
\text { and consolidated orders within the same time interval at } \mathrm{t} \text {-dpt time }\end{array}$ \\
\hline
\end{tabular}

The $\mathrm{Df}^{\mathrm{j}}\left(\mathrm{t}, \mathrm{t}_{0}, \mathrm{dpt}\right)$ allows to measure the reliability of the forecasts as soon as dpt goes to zero and $t$ approaches to $t_{0}$; these parameters represent a key performance about forecasts; vice versa $\mathrm{Da}^{\mathrm{j}}\left(\mathrm{t}, \mathrm{t}_{0}, \mathrm{dpt}\right)$ is a measure of the reliability of the orders provided by the different kinds of customers and allows to measure the related changes as soon as $\mathrm{t}$ approaches at $\mathrm{t}_{0}$.

\section{Conclusions}

This paper includes a description of a new business approach to develop slow food market in new regions through use of innovative supply chain models inspired to cloud manufacturing. Simulation is expected to be used in order to evaluate this new architecture as well as the adopted approach and criteria; this paper proposes a preliminary approach to the problem and it is devoted to create the framework to evaluate the reliability and consistency of the solution, as well as to identify alternative configurations and opportunities to improve the whole SCM performance.

The authors are currently cooperating for using this model in developing new business over Far East with products arriving mostly from South Europe and especially Italy. 


\section{Acknowledgment}

The authors are glad to thank Prof.Lin Zhang and his team from the Beihang University for being supportive in the development of this research and for sharing knowledge on CMfg.

\section{References}

1. Barroso, H.P., Machado, V.H. \& Machado, V.C. (2011) Supply Chain Resilience Using the Mapping Approach. Supply Chain Management, Pengzhong Li (Ed.)

2. Baruwa, O.T., Piera, M.À. (2008) "A derivative Control Mechanism for Supply Chain performance improvement",2008,"Proceedings of 22nd European Conference on Modelling and Simulation, Campora S.G., September

3. Bo Hu Li, L. Zhang, S. L. Wang, F. Tao, J. W. Cao, X. D. Jiang, X. Song, X. D. Chai. Cloud Manufacturing: A New Service-Oriented Networked Manufacturing Model, Computer Integrated Manufacturing Systems, 16(1), 2010, 1-8.

4. Bruzzone A.G., Massei M., Agresta M., Ferrando A. (2013) "Modelling Fresh Goods Supply Chain Contamination", Proc. of I3M, Athens, September

5. Bruzzone A.G., Longo F., (2012) "Simulation Based Analysis of a Manufacturing System devoted to Produce Hazelnut based Products", Proceedings of EMSS, Wien, September, pp 602-609

6. Bruzzone A.G., M. Massei, E. Bocca (2009) Fresh Food Supply Chain Simulation Based Case Studies in Logistic, pp. 127-146

7. Bruzzone A.G., A. Tremori (2008) Safety \& Security in retail: modeling value chain dynamics. Proc. of the 2008 Spring simulation multiconference San Diego, USA

8. Bruzzone A.G., S. Viazzo, F. Longo, E. Papoff, B.C., (2004) Simulation and Virtual Reality to Modelling Retail and Store Facilities Proc. of SCSC2004, San Josè, CA.

9. Busato P. R. Berruto (2009) Use of simulation models to study the dynamic of recall of non-conform perishable produce through the supply chain Int. European Forum on System Dynamics and Innovation in Food Network, Innsbruck-Igls, Austria

10. Christopher, M. and Peck, H. (2004) Building the resilient supply chain. International Journal of Logistics Management, 15(2), pp. 1-13

11. Cull, N. J. (2015) "Digesting the Milan Expo, 2015. Place Branding and Public Diplomacy, 11(3), 169-174. 
12. De Felice F., G. Di Bona, D. Falcone, A. Silvestri (2010) "New Reliability allocation methodology: the Integrated Factors Method", International Journal of Operations \& Quantitative Management Volume 16 Number 1, ISSN: 1082-1910

13. De Sensi, G., Longo, F., Mirabelli, G., 2008. Inventory policies analysis under demand patterns and lead times constraints in a real supply chain. International Journal of Production Research 46 (24) , 6997-7016

14. Dubeuf, J. P., Morand-Fehr, P., \& Rubino, R. (2004). Situation, changes and future of goat industry around the world. Small Ruminant Research, 51(2), 165-173.

15. Ernst \& Young (2011) "Capturing Recall Costs Measuring and Recovering the Losses", Grocery Manufactures Association (GMA),

16. Falasca M., Zobel, C.W., \& Cook, D. (2008) A Decision Support Framework to Assess Supply Chain Resilience, in the proceedings of the 5th International ISCRAM Conference Washington, DC, USA, May 2008, pp. 596-605.

17. F Tao, L Zhang, V C Venkatesh, Y Luo, and Y Cheng (2011) "Cloud manufacturing: a computing and service-oriented manufacturing model" Proceedings of the Institution of Mechanical Engineers, Part B: Journal of Engineering Manufacture, October 225: August 22 doi:10.1177/0954405411405575

18. Jüttner U., A. Ziegenbein (2009) Supply Chain Risk Management for Small and Medium-Sized Businesses Journal of Supply Chain Risk 124 pp. 199-217

19. Longo F., T. Ören, (2008) Supply chain vulnerability and resilience: a state of the art overview, Proc. of European Modeling \& Simulation Symposium, C.S. Giovanni, Italy

20. Hao Q, Shen W, Wang L. (2005) "Towards a cooperative distributed manufacturing management framework. Computers in Industry” 2005;56(1):71-84. [14]

21. Macías E.J., Mercedes Pérez de la Parte (2004) "Simulation and Optimization of Logistic and Production Systems Using Discrete and Continuous", Simulation 80(3): 143-152

22. Massei M. (2006) Logistics and process solution for supply chain of fresh food in retail, Proc. of MAS2006, Bergeggi October

23. Merkuryev Y., Galina Merkuryeva, Jonas Hatem, Bram Desmet (2008) "Supply Chain Simulation in the ECLIPS Project" Proceedings of the Second Asia International Conference on Modelling and Simulation, Kuala Lumpur, Malaysia, May, pp. 684-690.

24. Merkuryev Y., Galina Merkuryeva, Miquel Angel Piera, Antoni Guasch (2009) Simulation-based Case Studies in Logistics: Education and Applied Research. Springer-Verlag, London. 232 p. 
25. Merkuryeva G., Yuri Merkuryev, Hendrik Vanmaele (2011) "Simulation-Based Planning and Optimization in Multi-Echelon Supply Chains" Simulation: Transactions of The Society for Modeling and Simulation International. Vol. 87, No.8, August, pp. 698-713.

26. Montgomery D.C. (2000) "Design and Analysis of Experiments", John Wiley \& Sons, NYC

27. Parker N. (2007) "Intellectual property issues in joint ventures and collaborations" Journal of Intellectual Property Law \& Practice, 2(11):729-41.

28. Petrini C. (2013) "Slow Food Nation”, Rizzoli, Milan, Italy

29. Petrini, Carlo (2003) "Slow food: The case for taste", Columbia University Press, NYC

30. Pfohl H.C., H Kohler, D. Thomas (2010) State of the art in supply chain risk management research: empirical and conceptual findings and a roadmap for the implementation in practice Journal of Logistic Research 2(1) pp. 34-44

31. Portinari, F. (1989) “The Slow Food Manifesto”, Opéra Comique, Paris

32. Ren, L., Zhang, L., Wang, L., Tao, F., \& Chai, X. (2014) "Cloud manufacturing: key characteristics and applications", Int. Journal of Computer Integrated Manufacturing, 1-15.

33. Rodrigue J.P., C. Comitos, B. Slack (2006) The Geography of Transport Systems, Routledge

34. Rossi R., W.A. Rijpkema, J.G.A.J.Van der Vorst (2012) The impact of dual sourcing in food supply chain networks: the case of Egyptian strawberries, WICaNeM, Nerthelands

35. Sceffi Y. (2005) "Building a Resilient Supply Chain”, Harvard Business Review, 1 (8)

36. Sceffi Y., J. Rice (2005) A Supply Chain View of the Resilent Enterprise MITSloan Management Review, 47(1) pp. 41-48

37. Stravos T. P. (2012) Supply chain Resilience; Definition of Concept And Its Formative Elements Journal of Applied Business Research (28)5 pp. 921-930

38. Sun C., Yu H (2005) Supply Chain Contract under Product Cost Disrumption. Proc of the International Conference on Services Systems and Serivice Management, (1) pp. 708-711

39. Tao, F., Cheng, Y., Da Xu, L., Zhang, L., \& Li, B. H. (2014) "CCIoT-CMfg: cloud computing and internet of things-based cloud manufacturing service system", Industrial Informatics, IEEE Transactions on, 10(2), 1435-1442.

40. Vonolfen S., M. Affenzeller, A. Beham, E. Lengauer, S. Wagner (2011) "Simulation-based evolution of resupply and routing policies in rich vendor-managed inventory scenarios" Central European Journal of Operations Research, Vol. 19, No. 4, 2011, pp. 23

41. Wu D, Greer M.J, Rosen D.W, Schaefer D. (2013)“Cloud manufacturing: Strategic vision and state-of-the-art" J Manuf Syst http://dx.doi.org/10.1016/j.jmsy.2013.04.008 
42. Zhang, L., Luo, Y. L., Tao, F., Ren, L., \& Guo, H. (2010a) "Key technologies for the construction of manufacturing cloud. Computer Integrated Manufacturing Systems", 16(11), 2510-2520.

43. Zhang L, Guo H, Tao F, Luo YL, Si N. (2010b) "Flexible management of resource service composition in cloud manufacturing" In: IEEE international conference on industrial engineering \& engineering management. 2010. p. 2278-82

44. L. Zhang, YL Luo, F Tao, B. H. Li, L Ren, XS Zhang, H. Guo, Y Cheng, AR Hu. Cloud manufacturing: a new manufacturing paradigm $[\mathrm{J}]$. Enterprise Information Systems, May 21, 2012. (online published. DOI: 10.1080/17517575.2012.683812) 